années qui onl précédé la guerre, les 850.000 HP. qu'avec Ia collaboration des Pouvoirs publics, ils sont en train d'aménager depuis la guerre, vous sont un sùr garanl de la ténacité et de l'énergic avec laquelle les industricls de la houille blauche sauront, dans la France grandie, accomplir l'efforl nécessaire de demajn.

Commandant Henri Canres, Ancicn éleve de l'Ecole Polylechnique.

\section{L'ELECTROMÉTALLURGIE FRANGAISE}

\section{DES ALLIAGES FERRO-MÉTALLIQUES}

\section{RAPPORT AU $1^{\text {or }}$ CONGRES DU GENIE CIVIL}

par M. G. WIDMER, Secrétaire Général

de la Cue des Forges et Aciéries Electrioues Paul Girod

Nous avons montré, en de précédentes éludes, que les industries électrométallurgiques et électrochimiques sont celles qui, tout en participant à l'accroissement de l'activité industrielle du Pays, nous épargnent le plus de charbon, en nous donnant par.ailleurs, le moyen de faire rentrer de l'or par l'exportation à l'étranger de produits qui ne peuvent y être fabriqués aussi avantageusement. Ces fabrications sont, d'autre part, les plus grosses consommatrices de houille blanche et c'est sur leur développement que nous comptons pour la mise en valeur de nos richesses en forces hydrauliques. Il sest donc du plus haut intérêt de méditer les consi'dérations que développe le Rapport suivant sur la principale de ces industries, rédigé par un technicien d'une compétence notoire, et qui montre clairement l'œuvre à réaliser pour en assurer l'avenir. Nous invitons ă y collaborer tous nos lecteurs en situation de lui foumir un concours.

\section{LA SITUATION D'AVANT-GUERRE}

\section{Origine Frangaise de cetre récente Industrie}

Si l'on se propose tout d'abord de dégager les traits caracléristiques présentés avant la guerre par liélectromélallurgie des alliages ferro-métalliques, le premier qu'on lui reconnaîlra, non sans quelque fierté, c'est. une origine bien francaise.

Assurément, parmi tant de richesses dont la nature s'est plu à la favoriser, la France possède die larges ressources de forces hydrauliques ; mais encore convenait-il de trouve l'instrument qui permil de faire réaliser au courant électrique produil à l'aide de cette force un progrès de plus, et de transformer - au poinl de les ériger en une branche complètement nouvelle d'industrie - des fabrications encore embryonnaires à la fin du sièclé derniè.

On se souvient des produits spéciaux oblenus il y a vingt ans à peine au haut fourneau ou au cubilot : ce n'étaient à proprement parler, el en général péniblement élaborécs, que des "fontẹs spéciales ", à tencurs ẻlevées en carbore, en soufre, en phosphore et à teneur's Jimilées en élément spécial. Il a fallu le four électrique, qui, seul, constitue un laboratoire approprié à une juste proportion de la charge réductrice el à une température élévée, pour créer toute la variélé des alliages aujourd'hui indispensables aux aciéries comme' additions désoxydantes ou comme additions fixes et en vulgariser l'emploi par des prix réduits.
Et c'est précisément là qu'une fois encore s'est affirmé le giénie français.

Gràce, en effet, aux fours Héroult, Girod, Keller, Chaplel et similaires, - trop unversellement connus pour avoir besoin d'être à nouveau décrits, - la France, dès le début du $\mathrm{XX}^{\mathrm{a}}$ siècle, est devenue le pays classique du four électrique. Nombreux sont, en outre, les brevets d'adaptation à ce four du courant électrique "sous toutes ses formes enregistrés au nom des divenses sociélés qui, nées principalement dans les Alpes de la Savoie ou du Dauphiné, n'ont pas tardé à meltre en valeur le nouvel appareil.

Ces promoteurs français du four électrique ne se sont d'ailleurs pas contentés d'être des inventeurs et - par les procédés de fabrication qu'ils onl su appliquer - des métallurgistes : les grandes Aciéries, alors occupées à rechercher pour l'acier des qualités nouvelles par l'adjonction d'éléments nouveaux, n'ont pas eu dans celle voic de plus précieux collaborateurs et ce sont proprement leı.rs efforts commenciaux, tant en France qu'à l'étranger, ainsi qu'en fonl foi les archives de nos sociétés électrométallurgiques, qui ont eu le mérite de défricher et de créer le marché des ferro-alliages.

\section{Organisations symicales de Vente}

Ce marché d'abord ouvert à la libre concurrencs s'est peu à peu organisé, au moins pour les principaux alliages.

Avant la guerre, l'industrie électrométallurgique française des ferro-alliages comprenait douze sociétés (I). Certaines d'entre elles étaient entrées de plain-pied et sans antécedents dans la fabrication des ferro-alliages, et d'aulres - pour donner un emploi à une partie de leur force devénue disponible - y avaient été conduites soit par la crise qui sévit en rgoo sur le carbure de calcium, soit par les à-coups plus ou moins prononcés que celui-ci connut depuis lors.

En même temps, outre la Suisse, des pays tels que la Suède et la Norvègé complétaient l'équipement de leurs chutes, très régulières et extra-puissantes - partant, à courant si économique - en vue, principalement, d'entreprendre, sur une vaste échelle, la fabrication de l'alliage le plus consommé : le ferro-silicium, pour l'exportation duquel ils sont de plus si particulièrement favorisés.

Ainsi, la vulgarisation des procédés électrométallurgiques et l'orientation des capitaux étrangers vers les Pays scandinaves ne tardèrent-ils pas à faire naître pour les usines françaises de lourdes préoccupations et la concurrence générale tourna-t-elle rapidement à la lutte aiguë, avec ce carac-

(1) Ci-dessous la liste de ces soclétés, avec lindication de leur capital de l'époque, non compris les emprunts obligataires conlractés par plusieurs d'entre elles:

Soclété des Hauts Foumeaux et Forges d'Allevard, a

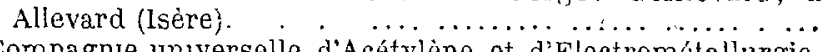
Compagnie umiverselle d'Acétylène et d'Flectrometallurgie. Les Clavaux, par Rioupéroux (Isère). . . ..... . ..... Société des Itablissements Veuve Charles Bertolus, 14, run

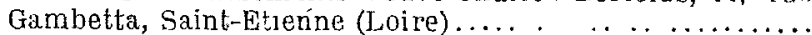
Compagnie générale d'Electro-Chimse de Bnzel, 22, rup de l'Alcade, Paris .......................... . Société des Carburès Méttalliques, 2 , rue Blanche, Paris... Socielé d'Blectro-Chmie, 2, rue Blanche, Parıs.. ....... Société Electro-Chımique du Gifre, Saint-Jeorve-en-Faucigny

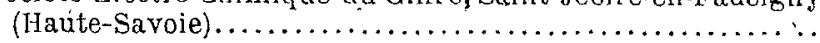
Société Electrométallurgique Procédes Paul Girod, Ugine

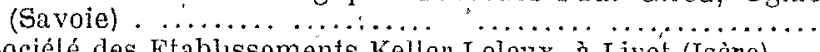
Sociélé des Fitablissements Keller-Leleux, à Livêt (Isère).... Société de Montricher, à Sant-Julien-de-Maurienne (Savole) Sociéte des Usines de Rioupéroux, à Rioupéroux (Isère) ... Société Electrométallurgique de Samt Béron, 37, rue de lá

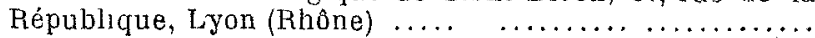

1.000000

3.800 .000

6.000 .000 3.300000 10000000 2.500000 12.0010 .000 3.250000 3.000 .00 . 2560.000

1.200 .000 
tère spécifique particulièrement grave que le développement des aménagements hydrauliques ayant été plus rapide que celui des applications qui pouvaient leur être données, les facultés d'absorption de la clientèle sur le marché des ferroalliages étaient insignifiantes par rapporl aux facultés de production des usines, si bien que, au moins pour les alliages formant le fonds commun, c'est-à-dire le ferro-silicium et le ferro-chrome, la nécessité d'une organisation proporlionnant les unes aux autres ne larda pas à s'imposer. Les services de ventes des diverses sociétés françaises furent donc mis en commun sous la forme de Comptoirs, Comptoirs internationaux, datant du début de rgr pour le ferro-silicium, et du début de $\lg _{2} 2$ pour le ferro-chrome.

Sans entrer ici dans les détails diorgánisation du Comptoir du Ferro-Silicium, par exemple, qui groupait vingt sociélés ayant leurs usines - neuf en France, cinq en Scandinavie, quatre en Suisse, deux en Italie et une en Autriche, - il est du plus haut intérêt et d'une cerlàine actualité puisque aussi bien certaines personnalités inexpertes se sont récemment appliquées à déformer et à obscurcir toute cette question - - de faire ressortir à nouvcau que, grâce à son avance en matière électromélallurgique, l'élément français y avait obtenu une participation, et, parlant, une influence morale que, ni l'importance de ses installations hydrauliques, ni l'importance de ses affaires antérieures, ni l'importance du marché national ne lui donnaient par eux-mêmes. Symbole de cet état de choses, le Comptoir avait son sìge, c'est-à-dire son centre de gravité, à Paris, alors que lo centre de la plus grosse production était en Scandinavie - par hégémonic hydraulique - et cclui de la plus grosse consommation en Allemagne - par vulgarisation, même pour la, fabrication des aciers communs, de l'emploi des alliages.

\section{Importation de Minerais. - Exportation de Produits}

Sous le couvert de ces oganisations syndicales et d'ententes individuelles entre certaines usines, l'industrie électrométallurgique française des ferro-alliages, importatrice des mincrais nécessaires à ses fabrications, élail exportatrice de ses propres produits.

Riche en chutes d'eau, la France - comme également - ses colonies - est, en effet, pauvre en mincrais autres que le fer et la bauxite ; les usines électrométallurgiques de ferroalliages devaient donc, avant la guerre, demander principalement :

Les minerais de chrome (en dehors̀ de' la Nouvelle-Calćdonie) à la Tunquic d'Asic, aux Tńdes el à la Rhodésie ;

Les minerais de manganèse (en dehors de quelques gisements français) à la Russic (Caucase) et aux Indes ;

Les' minerais de tungstène (en dehors de Puy-les-Vignes. (Haule-Vienne), scule mine française de l'époque) au Tonkin, au Portugal, au Siam, au Japon, à la Bolivie, à l'Arsentine, aux colonies anglaises telles que : les Indes, l'Australie, et surtoul les étals fédérés Malais (Straits Settlements) qui étaient' les grands pourvoyeurs ;

Les minerais de molybdène à l'Australie pour la molybdénite ; à l'Espagne pour la wulfénite.

Un certain nombre de ces minerais avaient, en réalité, deux lienx de vente : Jeur pays d'origine et.. Hambourg. Constatalion amère, mais qui trouvail un certain correctif dans le fait que les maisons avisées el très renscignécs sur le marché n'achetaient aux Comploirs allemands, toujours assez chargés et généralement disposés à l'allègement, qu'au moment où, sentant une certaine résistance dans les pays d'extraction, elles pressentaient une hausse. Ainsi, par un jeu de balance convenable, un équilibre régulatcur se trouvait-il maintenu.

Par contre, les ferro-alliages figuraient dans la balance du commerce de la France au titre des produits cxporlés. Sculs, en effet, en principe, le ferro-silicium à $10-1$ ? $\%$. Io silicospiegel et le ferro-manganèse qui, en raison de conditions de fabrication favorables sont restés du domaine du hautfourneau, étaient importés. Pour tous les autres alliages, nos sociétés électrométallurgiques, ñon seulement alimentaient exclusivement le marclié français, mais encore envoyaient au moins $80 \%$ de leur production à l'étranger ; savoir, en Angleterre, en Russic, en Italie, au Japon, dans certains cas même aux Etats-Unis, comme aussi cn Autriche et en Allemagne.

Vis-à-vis de l'Allemagne, le fail mérite d'ètre souligné, car pour tant de marchandises la France était tellement inondéc par la vague allemande qu'il n'est pas indifférent de signaIcr, quand on la rencontre, une catćgorie de produits qui remontait le courant.

Sans doute, l'Allemagne ne possède pas suffisamment en elle-même, faute de chutes convenables, les moyens de fabrication nécessaires, mais elle pouvait les rechencher ailleurs. Si elle ne l'avait pas fait, cela tenait à un véritable succès diplomatique du comptoir du ferro-sijicium ; par un marché, en effet, de 3.000 tonnes annuclles, conclu avec Krupp en février r9 ${ }^{2} 2$, pour la d'urée même de l'entente et, tout en laissanl une marge au profit des consommatcurs françáis, moyennant une bonification en sa faveur sur le prix des autres consommateurs allemands, - bonification tombée même à zéro en rgra par suite de la baisse des cours et marché dont toutes les livraisons furent exécutées par les usines non françaises de l'Entente - le Comptoir avait té assez habile pour conjurer le danger allemand. Ce danger allemand particulier consistait en ceci que Krupp, qui avait à sa disposition dans les Pays scandinaves des chutes d'eau importantes, dont les études étaienl tontes prêtes, n'avait qu'un mot à dire pour réaliser ces projets, non pas sur une petite échelle ou pour sa seule consommation, mais dans les proportions écrasantes que permettent les forces hydraulirues de. la Suède et de la Norvège. Ft, an vrai, de quoi s'agissait-il ? De cet alliage déprécié qu'était le ferrosilicium ? Sans doute : mais bien plus encore, derric̀re lui, de tous les aulres alliages qui étaient restés en majeure partie l'apanage de la France. Progressivement, mais fataleinent fabriqués par Krupp ol ses imitatcurs, coux-ci n'aurient pas tardé, à la manière allemande, à déferler $u r$ lo monde : l'œuvre régulatrice du Comploir aurail bicn en tendu élé balayćc par là-même pour faire place à la domination germano-scandinave ; mais, surtont, l'industric électrométallurgique française, réduite - ou à peu près au seul marché national, aurait ćté vaincue.

Dans le même ordre d'idées, une convention avait été conclue pour le ferro-chrome avec les fabricants américains de cet alliage.

Au total, en chiffres ronds ct approchés, on peut estimer que l'électrométallurgic française, avant ia guerre, importail pour six à sept millions de minerai, et exportail pour. une quinzaine de millions d'alliages.

\section{LA SITUATION ACTUELLE}

S'il a fidèlcment caractérisć l'électromélallurgie franģaisc des ferro-alliages avant la guerre, l'exposé qui précède aura laissé l'impression d'une situation cncorc avantageuse el 
prépondérante, mais pourtanl menacce ct fatalement destinée à s'aggraver.

Est survenue la guerre... Qu'en est-il résulté ?

Désorganisée toul d'abord, l'élcctromélallurgı - comme toutes les antres industries français's _ s'est ressaisie dès le jour oix, la lulle se propageant du thamp de balaille aux usines, il fut fait appel à son concours. Simple source d'approvisionnement des Aciéries ou des Fonderies, son rôle est plus modeste que colui dea usines d'où sort le natérieł de guerre : mais peu lui importe. Elle a conscience de remplir, au même titre que celles-ci, jusqu'aux limites des possibilités humaines, sa part de devoir national - et cela lui suffit !

Arant tout, elle n'a cessé d'assurer les besoins français qui n'ont cessć de croître.

On citera, à titre d'exemple, la production annuelle pour la consommation française - du ferro-silicium 25 à $90 \%$ qui est passéc prog̈ressivement de 3.000 lonnes calculées en $50 \%$ avant la gucre ì 9.000 tonnes actuellement - el cello du ferro-tung atène passéc environ de 250 à I. 800 lonnes.

Flle a pourvu igalement à unc large part des besoins de nos alliés, livrant particulièrement du frro-chrome à l'Angleterre, à la Rusise et à l'Ttalie, du ferro-tungstène et du ferro-molybdène ì la Russic et à l'Italie.

Pour parvenir à ces résultats, clle a su s'organiser, s'approvisionner, s'outiller.

Pour coordonner les demandes françaises el allices : unifier sa roprésentation' commerciale ol administrative auprès des Pouvoirs publics ef des industries privées ; en un mot, donner une formule à la solidarité dos divers intérĉts professionncls qu'nlle suscite, dle a consacró le système des Comptoirs, in reconstituant sur lo soul terrain français les Comptoirs du Ferro-Silicium et du Farro-Chrome, et en créant celui du Frro-Tunơstène ol du Ferro-Molybdène lous organismes dont la collaboralion éclairée a été appréciée cf utilisée par les services officiels.

De lour côté, an sein des difficultés communes des temps actuels, les efforts d'approvisionnement de l'Electrométallurgie ont été incessants, noțamment quant aux minerais. $A$ cet égard. elle a mis à profit de nouvelles sources productrices et ellc s'est intéressée - parfois pour des capitaúx importants - à certaines exploitations minières.

Pour le wolfram, en particulier, ce minerai que les Allemands en mal de tungstène, malgré les profits occultes de la contrebande, chargeaient leurs sous-marins dits commerciaux d'aller quérir ostensiblement, - elle a directement ou indirectement favorisé la mise en valeur en France de quatre nouveaux gisements, et travaillé - non sans peine, mais du moins avec fruit - le champ que lui ont dévoln les accords intervenus sur ce point entre les Gouvernements francais et anglais : le Tonkin, la Chine et la Corée, en particulier, se sont ainsi révélés producteurs' de wolfram, et des achats suivis ont été de même opérés en Espagne et aux Etats-Unis. - Parallèlement, la molybdénite rient aujourd'hui principalement du Canada.

Enfin, les usines électrométallurgiques se sont en même lemps adaptées aux circonstances, car auciune n'a hésité à procéder aux installations nouvelles nécessaires et plusieurs n'ont pas recilć devant ces travaux de longue haleine que sont les aménagements de chutes.

Pour embrasser l'effort d'ensemble accompli par l'Electrométallurgic française, il fandrail évoquer ici le dévclopement qu'elle s'est efforcée de donner aux diverses applica- tions du courant qu'elle a créé, telles que : distribution d'électricité aux villes ou aux grands centres industriels, fabrication de produits chimiques ou d'aluminium el service insigne par lès économies considérables de charbon ainsi réalisées - fabrication de l'acier el de la fonte synthétique. Mais ce tableau, où ne figureraient pas les moindres de ses étals de service, déborderait le sujet.

Si donc l'on se borne à rechercher une mesure de l'eflort réalisé dans la seule branche de l'électrométallurgic ici envisagée, on remarquera, cependant, qu'il est impossible de disinguer les ferro-alliages du carbure de calcium, car la plupart des usines poursuivent simultanément les deux fabrications. Pour les ferro-alliages, la production a, d'une manière générale, sensiblement suivi la demande ; par contre, il n'a pu en être toujours de même pour le carbure de calcium ; les besoins de ce produit inlermédiaire de la [abr]cation de la cyanamide sont devenus tels que les anciennes usines dé ferro-alliages qui n'en produisaient pas onl dù s'organiser à cet effel et que de nouveaux concours sont même devenus nécessaires - le toul ayanl pour objel de quadrupler la production d'avant-guerre ; d'où toute une noivelle série d'aménagements hydro-élcctriques en cours.

Au lotal, et bien qu'il soif en fait assez difficile de discerner la part de puissance consacrée à chacun des objets' des usines électrométallurgiques, on peul estimer approximativement à : $80.000 \mathrm{HP}$ moyens avant la guerre ; 'I50.000 HP' moyens actuellement ; 230.000 HP moyens après achèvement des travaux présentement en cours, la puissance consacrée à la fabrication réunie des alliages et du carbure de calcium dans les Alpes, cependant que 100.000 autres chevaux moyens environ sont, dans le même but, en aménagement dans les autres régions montagneuses de la France. 25.000 dans le Centre et 75.000 dans les Pyrénées.

Ces chiffres parlent d'eux-mêmes.

\section{L'État de cette Métallurgie a L'Étranger}

De leur côté, les pays étrangers n'étaient pas inactifs.

Nos alliés anglais ne trouvant guère chez eux les éléments d'une industrie électrométallurgique et privés par l'ouverture des hostilités de la poudre de tungstène (acide tungstique obtenu par précipitation chimique), qu'ils étaient habitués à recevoir d'Allemagne en ont, dès la première heure et, par un effort considérable, organisé de toutes pièces la fabrication dans leur pays $(x)$. Les deux principales Sociétés anglaises constituées à cet effet sont la Thermo Electric Ore Reduction Corporation $C^{\circ}$ et la High Speed Alloy's $C^{\circ}$, celte dernière n'étant en fait qu'un consortium formé par les trente plus importantes Aciéries de Sheffield.

L'apparition de ces entreprises a eu, pour première et immédiate conséquence, de faire naître entre la France et l'Angleterre un risque de concurrence sur le marché du minerai de tungstène : d'où ces accords qui ont réparti entre les deux pays les sources mondiales d'approvisionnement.L'Angleterre s'ćtant personnellement réservéc le minerai de ses colonies, la High Speed Alloys $C^{\circ}$ a pris des intérêts considé rables en Birmanie, notamment, dans ie district de Tavoy où le wolfram est de qualité supérieure, - district presque incónnu avant là guerre, mais'quj, grâce à l'appui du Gou' vernement britannique (constructions de routes, facilités de transport, recrutement de main d'cuvre chinoise) est de-

(1) L'Allemagne pour la fabrication des aciers rapides utilisait de préférence le ferro-tungstène dont elle avait reconnu les avantages et exportait principalement en Angleterre, où la tradition le maintient. presque tout le tungstène en poudre qu'elle produisait. 
venu actuellement un des centres les plus florissants de produclion du mincrai de wolfram.

Malgré sa situation privilégiée qui la met à même de recevoir de très importantes quanlités de minerai, celle Société ne parvient pas à utiliser le plein rendement de son usine.

Semblablement, l'Ang]elerie complète ses achats de forromolybdène aux Etats-Unis et au Canada par la fabricatiors chez elle de cel alliage. La Thermo Electric Ore Reduction Corporation $C^{\circ}$ de Lúton produit le ferro-molybdène au four électrique et The British Thermit Ltd de Liverpool par aluminothermie.

L'essor inouï donné par la guerre à l'industrie de l'acier aux Etats-Unis a, d'autre part, parallèlement développé d'une manière considérable dans ce pays la fabrication des ferro-alliages.

Les trois pays neutres détenteurs de puissance hydraulique : la Suisse et les Pays scandinaves, ces derniers riches - en Suède, d'environ 6.750.00o - et en Norvège, d'environ 7.50o.0oo chevaux hydrauliques, ont de leur côté donné à leurs installations hydro-électriques un développement intense.

Nous n'avons pu malheureusement rccueillir de chiffres précis à cet égard quant à la Suisse et à la Suède, mais on sait assez combien dans ce champ clos qu'est pour eux la Suisse, les belligérants se sont arraché toutes les ressources disponibles, pour être certains que le moindre kilowatl y a été employé. Nul doute, de même, que la Suède, où les Allemands possédaient déjà des intérêts avant la guerre, n'ait donné à l'utilisation possible đe ses chutes son plein développement.

Quant à la Norvège, nous avons eu la bonne fortune de recevoir d'une source officielle la statistique comparative suivante, singulièrement éloquente :

\begin{tabular}{|c|c|}
\hline$S$ DE & 1912 \\
\hline Ferro-Silicium" . & $6.022 \mathrm{~T}$. \\
\hline
\end{tabular}

Dans le même ordre d'idées, on signale, de source également sûre, le développement que tend à prendre en Norvège fá production des métaux destinés aux alliages pour aciers durs. Notamment une dizaine d'entreprises, en dehors des deux ou trois mines exploitées avant la guerre, se sont installées en Norvège pendant l'année $19 \mathrm{I} 6$, pour mettre en valeur des gisements de molybdène. En rgr4, la Norvège a produit 80 tonnes de molybdénite; en rgr5, 97 tonnes et en 1916 l'exportation en Allemagne seulement a été de 53 tonnes environ. Anglais, Suédois et Allemands s'intéressent à ces gisements.

On le voit - partoul; la métallurgic des alliages a, elle aușsi, tendu ses efforts au maximum.

\section{LA SITUATION D'APRÉS-GUERRE}

\section{La Production dépassant la Consómmation}

L'ensemble des faits qui viennent d'être rappelés forme si clairement démonstration que, avant même d'être consi gnée, la conclusion qui constitue comme la morale de ce rapport, se sera imposée d'elle-mệme à l'esprit : si elle n'en meurt pas, l'Electromélallurgie française des ferro-alliages sortira du moins de la guerre gravement touchée.

Devant le seul devoir de l'heure présente, elle ne sait acfuellcment que se réjouir des accroíssements qu'elle reçoit 1. ses adeptes anciens ou nouvcaux. Mais, qu'on ne s'y trompe pas, cette hypertrophie d'anjourd'hui n'est qu'une manière de suicide, tant clle développe l'organe au delà de la fonction normale de demain.

Un symptôme inquiétant annonce un autre mal qui aggrave encore le cas ; cest la tendance des grandes tríries à se rendre, pour la fabrication des alliages nécessaires a leurs besoins, indépendantes des usines élcctrométallurgiques. Soit directement dans ce but, soil dans celui plus large de ne pas tarder davantage à s'assurer une part dans

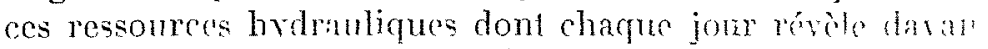
tage la valeur d'avenir, ces Sociétés puissantes acquièrenl. ou aménagent des usines hydro-électriques.

Du côté de l'étranger, l'horizon est, si possible, plun sombre encore - avant la guerre, l'Electrométallurgie françaisc des ferro-alliages, on s'en souvient, ne se défendail qu'avec peine contre l'ascension grandissante de la concurrence étrangère, Or, la gucrre, non seulement a anéanti d'un sen] coup les faibles remparts derrière lesquels notre industric électrométallurgique s'était retranchée, mais surlout - ol dans une proportion que les conceptions les plus hardice du temps de paix eussent laxée de folie — clle a mulliplić contre celle-ci le nombre des assaillants sur tons les marchés.

Abstraction faite de son cffort personnel, l'Anglcterre aurn appris le chemin de la Norvège dont les usines, déjà avantagées par le prix du courant, sont, pour ainsi dirc, à quai même : quel privilège pour l'exportation par rapport à la Savoie ou au Dauphiné ! I'Italis a. comme la France. pronfondément senti l'importance de ses ressources hỵdrantiques et elle est occupée à les mettre en valeur. Jeś EtatsUnis, plus encore que par le passé, se suffiront à etrx-mêmes s'ils ne deviennent définitivement exportateurs. If Japon même - on en a eu la preuve pour le ferro-tungstène - s'est partiellement outillé. Quant à l'Allemagne, elle sera désormais la cliente attitrée de la Suisse. de la Suède ét de l'Autriche, laquelle, grâce à une capacité de prod'uction supérieure à sa consommation, constituait déjì avant la guerre- - du moins pour les alliages courants - in marché ferme, vivant sur lui-même.

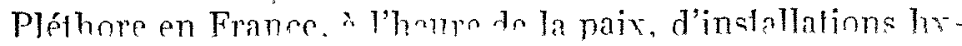
dro-électriques destinées à la fabrication des alliages of $\mathrm{dn}$ carbure de calcium ; - excès écrasant de la concurrence étrangère ; - comment devant de double phénnmène ́́co. nomique dû à la guerre, et en présence d'une consomma tion infiniment diminuée, l'Electrométallurgie des ferroalliages n'irait-elle pas au-devant d'une crise grave?

Si originellement, lors de la crise qu'il a traversée, le corbure de calcium a été secónru par les forro-alliages, - les deux fabrications étant depuis lors restées dans la plupart des usines le complément l'une de l'autre - ceux-ci pourront-ils à leur tour, dans les mêmes circonstances, recevoir de celui-là le même service? - Non, sans doute, car, on l'a vu, la multiplication des installations nouvelles - partant, de la concurrence - - se réalise surtout antuellement au profit du carbure, et de plus la gucre, en affectant la presque totalité du carbure disponible à la soudure autngène et à la cyanamide nécessaire au service dec poudres, a entraîné la suppression de la majeure narlie des installations d'acétylène pour éclairago créées par des efforte de plus do quinze ans. Après la gụerre, les annlications de l'acétylène et un peu de la cvanamide, rui cxige, erprndant, du carbure ì des prix très peu rémunérateurs, maintiendront bien. vraisemblablement, une ccrtaine vitalité à ce dernier, mais vitalité insuffisante à combler la défaillance des alliages ot neut-on vraiment espérer plus particulièrement que l'usage de la cyanamide se développera davantage dans l'agricul- 
ture, alors que, pour la seule fabrication des nitrates par extraction de l'azole de l'air, des aménagements importants de chutes sont actuellement en cour's en France mềme?

De nouveaux emplois pour les ferro-alliages, il n'en peut guère être attendu que du progrès au pas lent et incertain et de peu importants sans doule par rapport aux puissances disponibles. On se représente donc que la production des alliages, réduite à l'état de squelette, ne pourraiì plus être qu'une fabrication résiduelle, digne seulement d'absorber l'excédent de forces des hautes eaux ou les soldes de courant it que les usines électrométallurgiques de ferro-alliages et de carbure se trouveraient acculées à consacrer le plus clair de leur énergic à des objets plus rémunérateurs, parmi lesquels - placement de repos, mais déjà très concurrencé l'cxode du courant vers les centres industriels, même éloignés, ne serait probablement pas sans jouer un très grand role. Ainsi, l'Fleclromólallurgie française des ferro-alliages aurait-elle donné pendant la guerre son suprême effort et rommaîtrail-clle un déclin singulièroment prémaluré.

Pour se survivre, où l'Electrométallurgic française des ferro-alliages espère-t-clle donc trouver le salut?

Serait-ce, comme on le préconise pour d'autres indusiries, dans une certaine simplification de ses fabrications?

Bien que, dans une période difficile, telle que la guerre, il ait pu parfois paraîlre désirable, pour éviter la dispersion des efforts, de voir se concentrer sur quclques types judicicusement déterminés les demandes des Aciéries, il n'y a, cn temps normal, aucun bénéfice à réaliser par l'unification des alliages dans la consommation.

Bien mieux même, convient-il de laisser chaque fabricant d'acier utiliser les types les micux adaptés à ses spécialités ct de sauvegarder un des principes vitaux de l'industrie des alliages : celui d'une variété presque indéfinie, qui laisse libre carrière à linitiative simultanće de l'électrométallurgistes et de l'aciériste?

Tout au contraire, peut-on escompter un profit appréciable de la spécialisation dans la production? Les types d'alliages les plus consommés, tels que ceux du ferro-silicinm, du ferro-chrôme et du ferro-tungstène sont aujourd'hui suffisamment classés et limités pour permettre à chaque fabricant de se consacrer à une fabrication déterminée et suivie a l'cxclusion des autres - réalisation du travail en série, avec ses avantages classiques quant au prix de revient. Sur ce ierrain encore, les Compioirs auront un rôle des plus utiles à remplir car, chambres de compensation d'un genre spécial, c'est dans leur scin que s'élaborera entrc intéressés une juste répartition des spécialisations et que s'équilibreront les conventions financières qui en seront la conséquence.

Tea Sorićés élcolrométallurgiques de ferro-alliages ne failliront pas à ce programme, mais quel qu'en soit l'intérêt. le progrès susceptible d'être ainsi réalisé se présente comme si minime au regard de la portéc du problème'à résoudre que, pratiquement, il laisse celui-ci entier.

\section{Le Salut est dans l'Exportation}

Ancsi bion los meanres do salut sonterlles moins entre les sive ir nos Socintés électrométallurgiques qu'entre celles des Pouvoirs publics.

Un régime douanier adéquat à la situation, voilà, en effet, lc principal remède.

Avant la guerre, l'industric des forro-alliages - nous l'avons dit - vivait surtout d'exportation. el plus la guerre l'a développée, plus il est vrai que l'exportation peut scule lui conserver la vie. Ce dont il s'agit, par conscquent, c'ost bien moins de protéger le marché intéricur, qui doil évidemment lui rester acquis, que de lui rendre le marché extérieur qui est vilal pour dle. L'énoncé du dilemme es bien simple :

J'Flectrométallurgie irançaise des ferro-alliages sera exporlalrice ou elle ne sera pas.

Le régime douanier à établir entre alliés paraît légitimement dominé par ce principe qüil convient de prolonger sur le terrain économique l'alliance de Ja gucrre. Entre alliés, les échanges devraient donć s'opérer à parité, de lclle sorlc, notamment, que le tarif d'entrée en France d'un alliage venant, par exemple, des Etats-Uñis ou d'Italie, fût le mêmo que le tarif de ces pays à l'égard de l'imporlation françaiso re cet alliage.

Parmi les quelques pays curopéens ruslés noutres, la Suisse el les Etats scandinaves aturont été, pour une large part, pendant la guerre, les pourvoycurs de Pllemagne ch ferro-alliages. De plus, les capitaux allemands ont de lrès grands intérêts dans les usines de ces jays; ces inlérêls s'augmenteronl encorc de la nécessité, pour nos cnmemis, de changer de face et de prendre un masque pour essaycr d'échapper à l'hostilité ćconomique qu'ils onl dresséc cl accumulée contre eux : d'où la nécessité, à l'égard des neutres en généra], d'un tarif majoré sur le tarif minimum.

En outre, les pays alliés devront - comme pendant la guerre sur le lerrain militiirc - s'unir sur le terrain économique vis-à-vis des neutres, afin que ces neutres, ou éventuellement nos ennemis, no puissent róaliser des échanges au détriment d'aucun de ceux qui se seronl imposé las plus durs sacrifices de la guerre

On proposerait donc, en résumé :

$\mathrm{I}^{\circ}$.Entre pays alliés, un tarif préférentiel ;

$2^{\circ}$ Vis-à-vis des neutres, des tarifs moyens, résultant, après avis des Comptoirs intéressés et des Commission parlementaires des douanes, de l'application respective au tarif préférentiel ou a'u tarif général d'un coefficient d'augmentation ou de réduction déterminé, permettant, par conséquent, d'atteindre éventuellement ces derniers eux-mêmes: Ces tarifs moyens seraient essentiellemeni variables d'après les contingences relatives à chacun des pays neutres, telles rue questions politiques, tarifs de chemin de fer ou primes à l'exportation institués par ces pays, et autres éléments similairis (d'où la nécessité de jes rendre modifiables à toute époque moyennant un co'urf délai de préavis) et, en tout cas, présenteraient tous cctte condition que les produits qu'ils régissent devront avoir été intégralement fabriqués dans les pays neutres, et non pas' seulement achevés à la suite d'une demi-fabrication dans les pays ennemis ;

$3^{\circ}$ A l'égard des pays ennemis enfin, un tarif général offrant, par rapport au tarif préférentiel, un large écart par exemple, une différcnice du simple au double.

Par contre, et pour une duréc extrêmement longuc, permettant à l'équilibre européen de s'affermir sur les principes de paix définitive qui résulteront $\mathrm{du}$ grand conflit actuel - pour une durée de soixante-quinzc ì cent ans, par exemple - le traité de paix devrait imposer à nos ennemis, au profit des alliés, la clause de la nation la plus favorisée.

Incidemment, on ajoutera que lcs tarifs actucls relatifs aux ferro-alliages présentant des anomalies criantes et rcmontant à une époque où la fabrication de ceux-ci était encore établie sur des bases incertaines, ont le plus grand besoin d'être rajeunis. 
On.préconisera donc une nouvelle spécification soigneusemént étudiée et augmentée qui conservera comme base de taxation un classement des produits par catégorie, et dans chaque catégorie, par teneur de l'élément essentiel ; seuls, certains produits rares ou encore mal définis, dont le prix est susceptible de varier rapidement dans des proportions considérables et ceux qu'on attendrait de l'avenir sous la catégorie "d'alliages non dénommés " offriront une taxation ad valorem.

On voit immédiatement comment le projet de régime douanier qui vient d'être proposé - et qui laisse volontairement de côté toute question dowanière d'ordre purement théorique - présente en soi le double mérite d'être le plus conforme à l'équité générale comme le plus favorable à l'expansion des exportations d'alliages, puisque, en l'espèce, la plus importante concurrence provient de certains pays neutres - qui pourront partout être maîtrisés par des droits convenables - alors que la plus forte consommation reste celle de nos alliés enropéens - vis-à-vis desquels nous jouirons de droits préférentiels - et celle de nos ennemis, visà-vis de qui la victoire nous aura mis, commercialement parlant, sur le même pied que leurs meilleurs amis.

On n'a d'ailleurs pas entendu apporter ici une solution complàte et absolue du régime douanier à instituer pour les ferro-alliages. On a sevlement chorché à esmuisser ce système qui, créateur d'exportation, peut être complété par Inules autres dispositions ari londrainnl rscontinllement an même but, l'idéal restant que les Alliés, par des droits suffisamment protecteurs à l'égard de certains nentres, créent un véritablo marshé inlerallié conslitué de leur'a marchés rec. pectifs où ils s'accorderaient mutuellement la préférence

\section{Le Role de hal Législation FranciIse}

Pour s'aider elles-mêmes, en face du danger qui les menace, comme pour réaliser l'union trui fait la force et qui leur évite, notamment. d'être. du chef de leur clientèle, restreintes à nuelaues maisons. le iouet d'une mise en concurrence aussi facile que néfaste, nos sociétés électrométallurginues productrices do ferro-alliages persévéreront dans leur effort de groupement en Comptoir ; mais, dans cette voie, elles aimeraient sentir la terrain s'affermir doublement sous leurs pas.

Avant tout, la législation française de la matière devrait Atre remaniéé et nrécisée.

Réclamer, à cét égard, la modification de l'article fro du Code pénal ne sera pas un licu commun aussi banal qu'on pourrait le croire, car si dernièrement cet article, anrès avoir été l'un des nlus fossilisés de snn Code s'est vu misérablement exhumé et a trovvé un regain artificiel do vie. c'est à nos Snciétés électrométallurgioues - innocentes victimes de ses foudres - ru'il le doit. Pour autant, il n'a pas óté rajeuni, car - des industriole am iurieronsultes ot a" Gouvernement lui-même = on a été unanime à reconnaitrc m'il refardait. avañt ćlé concu nour 'une époque aussi éloionéc des conditions industrielles. commerciales et finanrières de notre sic̀cle aue les diligences d'alors le sont des chemins de fer d'aujourd'hui II a eu pourtant l'avantage d'ŝtre l'obiet de nombreuscá études $T_{\text {a }}$ dernière en date. dur. sous le natronace de l'Association Nationale d'Fxnansion Economique. ì la nlume hautement autorisée de MM De Segonne at Panl nf Rortsiers. semblè avoir définifivcrinent épuisé la auestion. C'est avec la conviction de lexpérience que l'on s'associera donc pleinement à leurs conclusions et que l'on souhaitera surtout de voir celles-ci, sans trop de retard, ratifiées par une loi.

A côté des traités officiels que représente le régime douanier, et pour en compléter l'effet, il peut se faire que l'industrie des ferro-alliages sente le besoin de composer son action avec celle de ses concurrents et soil, par là-même, conduite à passer avec certains de nos alliés, ou même avec certains neutres, des traités d'ordre privé.

Disons-le franchement: l'organisation bien comprise al si fondamentale de ses exportations - ou même celle de ses acquisitions de minerais - peut un jour l'entraîner à ressusciter la formule des Comptoirs internationaux de ferro-alliages. Comme M. Marcarve l'a fait à la tribune de la Chambre en février dernier, on demanderait donc, en second lieu, qu'une politique claire de principe fut adoptée à cet égard, et qu'il suffit, comme le déclarent MM. DE Segogne et Paul de Rousiers, de ne pas interdire les ententes inlernationales pour que celles qui poursuivent un but noloirement licite trouvent la protection légale à laquelle elles ont droit dans les seuls textes permettant les cntenles nationales. - Que s'il ne devait pas en êlre ainsi. il faudrait, on tont cas, ouc la question füt abordée de front et cur fuasent nettement définies les conditions lógales des cntentes induatrielles.

Le Comptoir est aujourd'hui une nécessité vitale pour toutes les industries d'une certaine importance. On ne compte plus les auteurs qui ont mis le fait en évidence en montrant. notamment, que le développement des cartels en Allemagne a constitué l'une des principales forces industrielles ef commerciales de ce pavs, ef ne sait-on nas de resle que. suprême évolution de ce mouvement. Ic Gomvernement allemand. nour les constituer en un front unique et continu en face de l'étranger, vient officiellement d'inviter les industriels allemands à se svndicuer. en leur laissant seulement le choix entre le svndicat rolontaire et le syndicat bligatoire?

Notre léniclation no saurnif done turdor davamlaon a so mettre à la hauteur des condifions économiques modernes et à donner à l'industriel. nar la connaissance rigoureuse. de l'étendue de ans droits. Ia tranquillité d'esprit dont il a besoin pour travailler

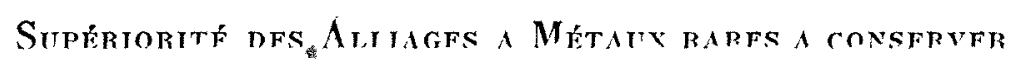

S'il est une catéơoric d'alliagrs où la France peut particurlièrement espérer conserver ses avantages. c'est assurément celle des alliaoss à mólaux rares Une des nrincinales nréoccumations de lindustrie do ces nroduits restant cello des approvisionnements en minerais anrès la guerre. trois verux à cet égard se recommandent ì l'attention.

Par une internrétation laron do Ta résolution de la Confóronce économiane des Alliḱs nar lammelle renx-ci so sont díclarés d'accord nowr réserver aux nave alliós avant tons' aufres. leurs ressources naturelles nendant la nériode de resfauration. l'Electrométallurgie francaise des ferro-alliages voudrait le marché mondial dos minoraic snóniavr totalo

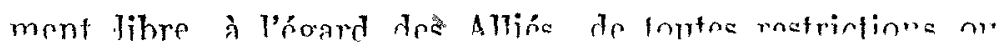
charoes d'exnortafinn

Des pays riches en wolfram, tels que les Colonies britannicurs ou los Fiats-Unis, auront toujours une propension fiscale naturelle ì frapper la sortic de ces minerais, comme le fait déjà le Portugal, d'une taxe.d'exportation. Ils v ceront, en outre, poussés à la fois par les producteurs de minerais - qui auront su concentrer entre leurs mains un noyau de mines puissantes - et par leurs fabricants nationaux 
de ferro-tungstène, qui auront à supporter, après la guerre, la réduction de la consommation. Pareilles charges, si elles se développent, ne deviont frapper que les pays ennemis ou neutres.

On souhaiterait, d'autre part, que les minerais dont a besoin l'Electronétallurgic des alliages, fussent introduits en France par importation directe. Il serait essentiel de ne plus voir les minerais d'oulre-mer draînés vers les ports allemạnds, comme ils l'étaicnt avant la guerrc, ou vers les ports anglais, comme ils le sont depuis la guerre, pour êlre dirigés ensuile sur la France, qui paic ainsi, outre la surtaxe d'entrepol, tous les frais d'un double trafic maritime factice. En allendant que les armateurs français soient en possession des navires qui affranchiront la France du fret ćlranger, l'organisation d'escales dans nos ports des bateaux ctrangers s'impose.

Fnfin, pour faciliter efficacement les rapports entre- les achetcurs français et les producteurs étrangers, on saluerail avec une satisfaction à la fois patriotique et professionnelle la création d'une Banque des métaux à l'instar, il faut bien I'avouer, moins cependant l'esprit de conquêtc, de la Metallbank et de la Maison Beer, Sondheimer et $C^{\circ}$ de Francfort.

On concevrait une telle banque fortement organisée sur les principes modernes.

Négociante elle-mème en minerais, elle en controblerail lo marché. Elle pourrait, en tout temps, renseigner les intéressés sur la valeur de tout minerai et constituer à Paris, où il n’en existe pas, un centre de cotation qui serait à dével'pper cl une source sîro do statistique. Elle serait en rapport, dans le monde entier, avec des échantillonneurs sérieux ef des chimistes capables, pour permettre de traiter les affaires à distance. S'i] se manifeste ajourd'hui une certaine tendance à l'établissement d'usines de produits finis dans les lieux même de provenance des minerais - tel est notamment le cas en. Australie - il serait de son ressort d'enrayer cé mouvement el de contribuer à attirer le mineraj en Europe.

Elle" se tiendrait, d'autre part, à l'affût des affaires minières et, capable de les étudier d'une façon pratique, clle serail en mesure de les proposer utilement à ses adhérents. Elle possćderait à cet effet des représentants ayant des conceptions commerciales robustes et ne craignant pas l'efforl, qui constitueraient en quelque sorte son état-major consuJaire. Pour l'ensemble, elle présenterail une organisation bancairc souple et économirque, recourant largement, en particulier pour les paicments, au sýstème des virements.

Qui ne voit qu'une telle banque, en état de se mesurer avec toutes les concurrences, remplirait un ròle commercial et nalional des plus féconds ?

\section{La Législation des forices hydrauliques}

L'électrométallurgie est trop étroitement intéressée à la législation des forces hydrauliques pour que l'on ne s'arrête pas un instant ici à cette question. Aussi bien, est-elle toutc d'actualité.

Dans les divers pays délcnteurs de réserves hydrauliques, la crise du charbon a tourné les ycux vers l'énergie contenue dans les chules d'ean et orienté les esprits vers l'organisation rationnelle de ces fonces. La Suisse, Ia première, vient de promulguer une loi sur cet objet.

En France, des projets d'origines diverses ont été élaborés. Finalment, pour aboutir promptement, comme le réclame. l'opinion publique; une Commission extra-parlementaire a été instituée en .mai IgI7 et celle-ci a rapidment mené sa làche à bien.

Le régime des riverainetés - découlant d'un code pour qui'la propriété privée, avec son caractère de perpétuité, est un dogme - est définitivement abandonné.

A cetle occasion el au lerme de sa carrière, ompressonsnous de le saluer avec reconnaissance dans l'éclosion romarquable qu'il a su, fócondé par l'initiative individuelle, donner à l'appropriation en France de l'énergie hydraulique.

Il sera désormais remplacé par celui de la concession émanant de l'Etat. L'Etal, dispensateur de l'énergie contenue dans les cours d'eau, en atlendant qu'il le soit de celle des marées, n'en remet l'usage à des mains privécs que pour un temps et sous des conditions déterminées, mais il confère en contre-partie au concessionnaire des prérogatives propres à faciliter les aménagements de chutes ; puisse-t-j] en même temps conférer l'esprit de célérité à ceux qui, próposés au sort des formalités nouvelles, seront, pour chaque aménagement, les maîtres de l'heure !

Le projel de loi relatif à l'utilisation de l'énergie hydraulique, que la Commission a rédigé, a rallié l'unanimité de ses membres, parmi lesquels l'industrie électroméfallurgique comptait ses représentants les plus autorisés. G'est dirc que cette industrie ne saurait s'opposer audit projet, s'offrant, après s'être édifiée à l'aide des principes du Codc civil, à accepter les temps nouveaux. Elle en souhaite donc le vote - sans restrictions ni innovations - suivant les principes qu'il pose, en nourrissant en outre l'espoir que pour éviter une aventure semblable à celle dans laquelle la guerre a surpris les mines, c'est-à-dire une période intermédiaire frappée de stérilité complète, la législation actuelle ne s'éteigne pas facticement avant l'instauration légale du nouveau régime.

Grande consommatrice de matériel électrique, l'électrométallurgie des alliages était bien placée avant la guerre pour connaître les offres avantageuses a'u point de vue commercial et technique des constructeurs étrangers.

Par esprit de solidarité nationale, elle ne désire rien tant que de réserver ses ordres aux maisons françaises. Celles-ci sont les premières à avoir conscience de l'effort à réaliser, tant par elles-mêmes que par les banques d'Etat pour se mettre au niveau des cinconstances. L'électrómétallurgie suivra cet effort avec la plus grande sympathie et le favo. risera de tout son pouvoir.

\section{Nécessite de l'Ápplication des Lois sociates}

On ne s'est attaché, jusqu'à présent, qu'aux problèmes touchant directement l'Electrométallurgie des ferro-alliages. On voudrait, en terminant, s'intéresser rapidement à quelques-unes des grandes questions que soulève la reconstruction industrielle de la France, en s'assocjant, d'une manière générale, aux conclusions que les spécialistes de pareils sujets auront dégagées de leurs ẻtudes d'ensemble.

On n'hésitera pas à proclamer, avec toute l'industrie et l'immense majorité de nos concitoyens, que la politique pure a surabondamment démontré sa stérilité.

Notre victoire militaire, quelle qu'en soit la gloire. ne sera qu'une préface : c'est notre victnire économique qui écrira la page définitive, celle dont noùs demanderont comnte les générations futures. Donc, plus de programmes théoriques de partis, plus de vains sophismes de réunion publique. Poür tous les Français, électeurs et élus, il n'y a qu'un devoir 'et il est assez vaste : travailler an développement de l'áctivité économiquè du pays. 
Le problème de la main-d'œuvre, qui sera, sans doute, un des plus délicats de l'après-guerre, puisqu'il devenait déjà préoccupant auparavant, pose ceux de la dépopulation el de l'emploi des femmes, qui sont d'ajlleurs intimement liés.

Tous les appels qui seront adressés à l'industric pour étüdier ces problèmes ou en favoriser la soTution, l'Electromélallurgie est d'ores et déjà prête à y répondre.

Personnellement, l'industrie électrométallurgique, eu égar. à l'imporlance de sa produclion, occupe relativement peu de main-d'œuvre et, par le développement rationnel d. mécanisme, notamment dans les travaux de manutention, clle pourrait encore réduire son personnel. Certains de ses emplois peuvent êlre confiés aux femmes.

Généralement situées loin des villes ct isolées par nature, nos usines électrométallurgiques sont ordinairement complélées par des cités ouvriêres, avec, parfois, des écoles pour les enfants.

Quant à l'alcoolisme, on déclarera très neltement qu'il axiste un remède contre ce fléau, mais l'Electromélallurgie ne connaît que celui-là : c'est la suppression radicale de l'alcool non industriel.

Des lois ouvrières ou sociales existent, d'autres sont à créer. Mais le plus nécessaire serail d'assurer leur applicalion. Ce n'est, malheureusement, pas le cas dans bien des circonstances.

Des Electrométallurgistes ayant subventionné des écoles, se plaignent de ce que beaucoup d'enfails ne suivent pas les classes el que, pourtant, on n'a jamais vu l'Administration sévir à l'égard des parents pour inobservation avérée de l'obligation scolaire.

On connaît semblablement l'échec qu'a éprouvé, du fait de l'indifférence de ses bénéficiaircs, la loi sur les retraites ouvrières. On sait l'effort de l'Administration pour l'introduire dans les usines à la faveur de la mobilisation, mais ce n'est là qu'un expédient. Ou bien il faut apporter à l'observation de cette loi toute l'énergie voulue, ou bien, si elle est réellement inapplicable, 'la modifier, mais ce qui est essentiel, c'est qu'elle soit appliquée ; lcar, il est pénible, pour les rapports entre ouvriers et chefs d'industrie, de rencontrer de pareilles lacunes, qui sont susceptibles de créer des malentendus regrettables.

En raison de la situation précaire dans laquelle la laissera la guerre, il ne faudrait frapper l'Electrométallurgie des ferro-alliages d'impôts que d'une main légère. Sa part contributive croîtra avec sa prospérité, qui constitue le véritable fonds où l'Etat pourra puiser. Dans la mesure de ses facultés, l'Electrométallurgie des ferro-alliages prendra donc sa part des lourdes charges d'après-guerre; mais, comme les autres industries, elle in'accueillera qu'avec réserve tout système d'imposition qui permettrait au fisc l'intrusion dans ses affaires.

Bien qu'industrie à capitaux d'importance moyenne, l'Electrométallurgie des alliages n'a généralement trouvé d'appui véritable qu'auprès des banques locales. Dans le même temps, au plus grand détriment de l'industrie française, les grands établissements de crédit se répandaient en 'missions - canal néfaste d'exportation des capitaux français à l'étranger. Tout a été dit sur ces méthodes de crédit d'avant-guerre ; presque tout également sur les réformes ru'elles réclament. On souscrira des déux mains à ces études compétentes.

Lie Role des Ecoles Techniques et Professionnelles

L'Elcctrométallurgie, qui a besoin de spécialisics, n'est pas rcslée inäifférente à la vaste cnquête récemment ou- verte sur la formation des ingénieurs el le rôle des.écoles lechniques ou professionnelles.

Peut être est-ce une erreur pour cerlaines écoles de sélectionner les intéressés à l'entréc el serait-il prélérable en ouvrant largement la porte des ensergnements spéciaux - à l'instar des Universités - de ne placer le concours qu'exclusivement au terme des cycies d'étude.

S'il en était besoin, l'exemple, pendanl la guerre, de l'Allemagne, qui, sans ses savants," cût été plus rapidemont perdue, a trop démontré l'utilité do la collaboration étroite de la science et de l'Industrie, pour qu'on ne désire pas, surlout dans wne industrie qui ne fait quappliquex en grand des procédés de laboratoire, voir se créer, entro le savant et l'industriel, une intimité qu'exchanl jusqu'ici une mentalité un peu différente. Tous deux se rencontreraicnt ulilement à l'usine, mais c'est surtout au laboratoire que l'urs efforts mis en commun deviendraicul féconds. Combien seraient donc les bienvenus ces laboratoires de recherches qui, exclusivement orienlés ver l'étude de procédés pratiques, constitueraient comme l'avant-gardo de l'industric. Apres avoir largement doté de telles inslitutions on les pourvoyant de tous les instruments de travail dont elles auraicht besoin, l'industrie ne devrail pas hésiter ì fairo récolter au savant qui lui aurait ainsi fait r'óaliser des progrès des avantages matériels en rappor't avec ceur qu'il l'u' aurait procurés.

\section{Améliorations désmables d'Ordre commercial}

Importatrice de minerais et exportatrice d'aliiages, l'industrie électrométallurgique des alliages a un intérêt de premier ordre à la rénovation de la marinc marchande, d̀ l'agrandissement de nos ports lamentablenent en retard; bref, à toutes les mesures qui rendront au pavillon et au commerce maritime français la place qu'ils devraient posséder et permettront à nos usines - tributaires de l'étranger pour les transports sur mer - de trouver enfin du fret national en rapport avec celui de la eoncurrence. Quel pém nible souvenir les électrométallurgistes ne conservent-ils pas de l'avant-guerre où les frets français coụtaient quatre ou cinq fois plus cher que ceux qui se rallachaient aux ports d'Anvers et de Hambourg !

Au même titre, nos sociétés électromélallurgiques s inscrivent, elles aussi, contre l'organisation actuclle de notre corps consulaire qui est à reprendre de fond en conble et à laquelle il est urgent de substituer une représentation puissante et compétente, sur les bases essentielles de laquelle tous les intéressés soni dès maintenani d'accord.

Généralement situées dans des replis isolés de momagnes, on marge de voies de communıcations à grand trafic, les usines électrométallurgiques prennent un intérêt tout particulier, pour la rapidité de leurs relatións extérieures, aux projets d'amélioration des services postaux, télégraphiques et téléphoniques qui sont l'objel des préoccupations du ministère du Commerce. Elles se féliciteraient du prompt aboutissement de ces projets conçus dons l'esprit le plus heureux, puisqu'ils envisagent l'autonomie administrative des dits services et leur exploitation suivant les principes industriels.

En fixant la pensée sur quelques-uns des grands problèmes que léguera la guerre - el combien d'autres solliciteraient encore l'intérêt - on a surtout entendu témoigner de ce que rien dans le grand labeur de la paix recourvée ne sera étranger à l'Electrométallurgie. 
Il ne faut cependant pas que l'ampleur des horizons qu'on vient d'entrevoir distraie l'attention de l'objet spécial du présent rapport.

Née de la terre et du génie français, l'Electrométallurgie des ferro-alliages avait déjà connu, avant la guerre, la menace d'une concurrence plus puissante qu'elle; mais elle avait su momentanément l'enrayer.

La guerre, en intensifiant l'Electrométallurgie universelle, a rapproché et accru le danger.

Contre celui-ci, nos sociétés électrométallurgiques lendront assurément toute leur énergie et sauront notamment trouver dans leurs groupements - surtout si les fondements de ceux-ci sont légalement affermis - des moyens de résistance.

Mais, qu'on en soit bien convaincu, le vérilable secours leur est extérieur : il ne peut venir que d'une organisation douanière génératrice d'exportation.

En livranl cclte conclusion fondamentale à la conscience des Pouvoirs publics, on voudrait surtout les voir partager le sentiment élevé qui a présidé à l'élaboration de ce rapport ; car, si le dévoucment professionnel n'a évidemment pas laissé de l'inspirer, une conviction plus profonde encore n'a cessé d'en dominer l'ensemble : celle de conserver à la France, qui a besoin de toutes ses énergies, une industrie lui appartenant à tant de titres.

En résumŕ, le Congrès pourrait émetre ces voux spéciaux:

\section{VCEUX SPÉCIAUX}

ler Vøu. - L'Electrométallurgie' française des ferro-alliages, trouvant dans l'exportation son principal et plus essentiel débouché, émet le vou que le régime douanier qui sera institué à l'issue de la guerre soit établi :

I $^{\circ}$ Entre les Alliés, sur les bases d'une fédération créant, à l'aide de leurs marchés respectifs où ils s'accorderaient mutuellement la préférence, un marché interallié dûment prolégé — bien qu'à des degrés divers — contre les nations neutres ou ennemies;

$2^{\circ}$ Vis-à-vis des pays ennemis, avec le bénéfice de la clause de la nalion la plus favorisée, sans réciprocité si possible.

2e Vav. - L'Electrométallurgie française des ferro-alliages émet le vou que l'arlicle 419 du Code pénal soit modifié.

3. Vav. - L'Electromélallurgie française des ferro-alliages émet le voeu que, par une application particulière de la fédéralion douanière désirable entre Allés, les minerais deslinés aux Alliés ne soient pas frappés de droils de sortie par les pays producteurs de ceux-ci.

De mêmé, clle désire ardemment que ces minerais soient introduits en France paŕ importation dirccte, c'est-à-dire sans transil intermédiaire dans d'autres pays et de préférence sous pavillon français, ou, à défaul, par escales dans les ports français de balcaux étrangers.

$4^{\circ} V_{\alpha U}$. - L'Electrométallurgie française des ferro-alliages émet le vou qu'il se constitue à Paris un centre d'achat de minerais avec tous les organes correspondants et, qu'en particulier, une cote des minerais soit créée à Paris.

\section{LA PRODUCTION DE L'ALUMINIUM AUX ĖTATS-UNIS}

Jo prix de l'aluminium vient d'être fixé par le Gouvernemient et des statistiques ont été dressées pour faire connaître le développement de cette nouvelle industrie. Sui- vant les renseignements donnés par la "National City Bank " de New-York, la production de l'aluminium s'élevai! de 60.000 livres en 1890 à 7.000 .000 de livres en I9oo, pour atteindre 48.000 .000 en rgro, 100.000 .000 de livres en rgr5 et I80.000.000 de livres en I9I7. Les bénéfices onl augmenté dans les mêmes proportions, bien que le prix d'achat ait diminué et la production de ce métal qui rapportail, els Igoo, environ deux milions de dollars, a donné, en I9I7, près de $\$ 46.000 .000$. Entre 1944 et rgr $_{7} ;$ ' les exportations de l'aluminium ont augmenté aussi. de \$1.047.000 à \$20.300.000. Les Etats-Unis, grâce'à la guerre européenne, ont obtenu la première place dans cette production, commc dans beaucoup d'autres. Ils fondent, en effet, près de la moitié de ce qui se manufacture dans le monde, soit de 135.000 à 550.000 lonnes, suivant les slatisliques.

Ge qui a donné un grand essor aux usincs qui traitenl ce produit, c'est l'emploi des fours électriques Héroull ol la substitution de l'aluminium à l'étain dans divers cas. De plus, son prix n'est pas supérieur à celui de l'étain el l'approvisionnement n'est pas menacé par les sous-marins, car on trouve son mincrai en grande quantité dans différents Etats de l'Union. En rgr7, les Etats-Unis ont importé pour $\$ 65$ ooo.00o d'étain de la Péninsule malaise, des Indes hollandaises et de Bolivie. C'est donc avec une grande satisfaction que les Américains déclarent qu'ils espèrent se servir d'aluminium au lieu d'étain dans la fabrieation de leurs aéroplanes, quand ils arriveront à la mettre au point. Les journaux du pays se plaignent et, pour leur donner satisfaction, on a nommé trois ou quatre commissions qui vonl rechercher qui est responsable des retards qu'on constate el pourquoi, après avoir dépensé beaucoup d'argent, on ne montre pas des résultats très appréciables.

La production de l'aluminium dans le monde a été, "dit-on, d'environ r/40.000 tonnes en $\mathrm{xg} \mathbf{6} 6$, dont la part des EtalsUnis a atteint 62.00o tonnes; celle de la France, 20.000; de la Norwège r6.000 et du Canada, 8.00o. Les renseignements sur cette production pour la GrándeBretagne diffèrent considérablement, car on parle de 4.000 tonnes et certains indiquent i 2.000 tonnes. En Suisse, le rendement normal est de 20.000 tonnes, mais il a été réduit, parce que - les usines n'ont pas pu recevoir facilement toute la beauxilé que leur fournissait la France. Entre parenthèses, nous ne voyons pas bien pourquoi nous livrerions notre minerai dumineux à la Suisse qui est trop près de l'Allemagne et de l'Autriche. Il ne faut pas oublier 'qu'une opération du genre de celle des carbures peut se répéter pour d'autres produits indispensables ou même utiles à nos ennemis.

La beauxite contient, tout le monde le sait, l'alumine d'où le métal est retiré et on en trouve dans divers territoires de l'Union, mais la plus grande quantilé provient de l'Arkansas et les plus importantes usines qui emploient ce minerai se trouvent près des chutes du Niagara. D'autres fabriques se sont installées dans les Etats de New-York, de Pensylvanie, de New Jersey, de Maryland, du Tennessee et de la Caroline du Nord. Comme il a été dit plus hauth, le prix de l'aluminium a grandement changé depuis l'éporue où a commencé sa fabrication commerciale, en 1870 . Ce prix était de $\$ r$ la livre et en $r 88 \mathrm{~g}$ il avait baissé à \$2 oo, pour descendre encore a 33 cents. nuis ì ? ? cenls en rọ 3. La demande de quantités considérables ei l'angmentation des salaires et des frais de fabrication, l'ont reporté à 33 cents, et le Président vient d'en fixer le prix à 32 cents, pour la période finissant au ${ }^{\text {er }}$ juin $\mathrm{r} 9 \mathrm{r} 8$. Le prix de l'étain est, en moyenne, de 43 cents, soit à cause de 